\title{
INDICATORS OF PARAMEDIC SERVICE USE BY COMMUNITY DWELLING OLDER ADULTS
}

\author{
Matthew S. Leyenaar $\mathrm{MA} P h D(c)^{1^{*}}$, Walter Tavares $\mathrm{PhD}^{2,3}$, Gina Agarwal MBBS \\ $\mathrm{PhD}^{1,4}$, Andrew P. Costa PhD ${ }^{1}$ \\ *leyenam@mcmaster.ca
}

\section{OPEN ACCESS ARTICLE}

Recommended Citation: Leyenaar MS, Tavares W, Agarwal G, Costa AP. Indicators of paramedic service use by community dwelling older adults. Irish Journal of Paramedicine. 4(1). Jun 2019 https://doi.org/10.32378/ijp.v4i1.74

Received: 28 Feb 2018

Revised: 25 Oct 2018

Accepted: 13 Dec 2018

Published: 19 Feb 2019

Copyright: (c) 2019, the authors. This is an Open Access article distributed under the terms of the Creative Commons Attribution-Non-CommercialShare Alike 4.0 International licence which permits use, distribution, and reproduction in any medium, provided the original work and any attributes thereof are properly cited, are distributed under the same licence, and that the work is not used for commercial purposes.

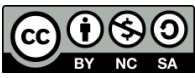

Funding/support: Canadian Frailty Network (ML)

Competing interests: None declared.

Provenance and review: Not commissioned peer-reviewed.

\section{Author affiliations}

1. Department of Health Research Methods,

Evidence, and Impact, McMaster University,

Ontario, Canada

2. Division of Emergency Medicine, Department of Medicine, McMaster University, Ontario, Canada

3. Faculty of Medicine, University of Toronto,

Ontario, Canada

4. Department of Family Medicine, McMaster

University, Ontario, Canada

\section{Acknowledgments}

The authors wish to thank Jane Blums and Dilys Haughton at the former CCAC for their support.

\section{Abstract}

\section{Introduction}

Home care clients represent a patient group that may be served through community paramedicine (CP) programs. The Detection of Indicators and Vulnerabilities for Emergency Room Trips (DIVERT) scale was recently validated to identify levels of risk for use of emergency care among this population. The objective of our study was to investigate whether home care clients that were identified as being at higher risk based on their DIVERT scores were more likely to use paramedic services to access the emergency department within 90 days of assessment when compared to clients that had lower DIVERT scores.

\section{Methods}

A retrospective cohort study was conducted using regularly collected administrative data. Home care assessment data were supplemented with data on emergency department (ED) visits. Arrival by ambulance was modelled to control for DIVERT scores as well as several social and demographic variables.

\section{Results}

Within the cohort, approximately $40 \%$ of individuals visited an ED within 90 days of a home care assessment and almost half of all individuals visited an ED more than once within a year. About two-thirds of clients that visited an ED in the 90 days following assessment used an ambulance for transportation. DIVERT scores were predictive of paramedic service use with highest scores indicating 4.15 times higher odds of arriving at the ED by ambulance (95\% $\mathrm{Cl} 3.60-4.78)$.

\section{Conclusion}

The results indicate that the DIVERT scale can be used to identify community dwelling older adults that are likely to use paramedic services to take them to the ED. Further investigation of aspects of social isolation, carer resiliency, time of use, and characteristics associated with ED discharge are warranted. Using the DIVERT scale as a case-finding tool may help community paramedicine and home care agencies improve care for frequent ambulance users among this population.

Keywords: ambulance utilisation; patient-care management; risk factors; home care services; community paramedicine 


\section{Introduction}

The task of case-finding patients at risk of using the emergency department (ED) includes the challenge of considering interactions between multiple disease processes.(1) A case-finding tool was recently developed and validated to identify levels of risk of ED use for home care patients.(2) The Detection of Indicators and Vulnerabilities for Emergency Room Trips (DIVERT) scale generates six levels of risk for ED visits-from one (lowest score) to six (highest score) - following a standardized home care assessment.(2) Scores are determined by a variety of factors including past use, clinical diagnoses, key interventions, and other assessment findings (see Figure 1).(2) The performance of the DIVERT scale as a pre-emptive case finding tool is driven in part by community dwelling older adults' history of emergency department visits and hospital admission.(2) It differs from other predictive tools by using ongoing symptoms and health conditions to differentiate levels of risk.(2) While DIVERT scores have been effective in identifying patients at risk of ED visits, the role of paramedic services in facilitating transport for these patients has not been investigated.

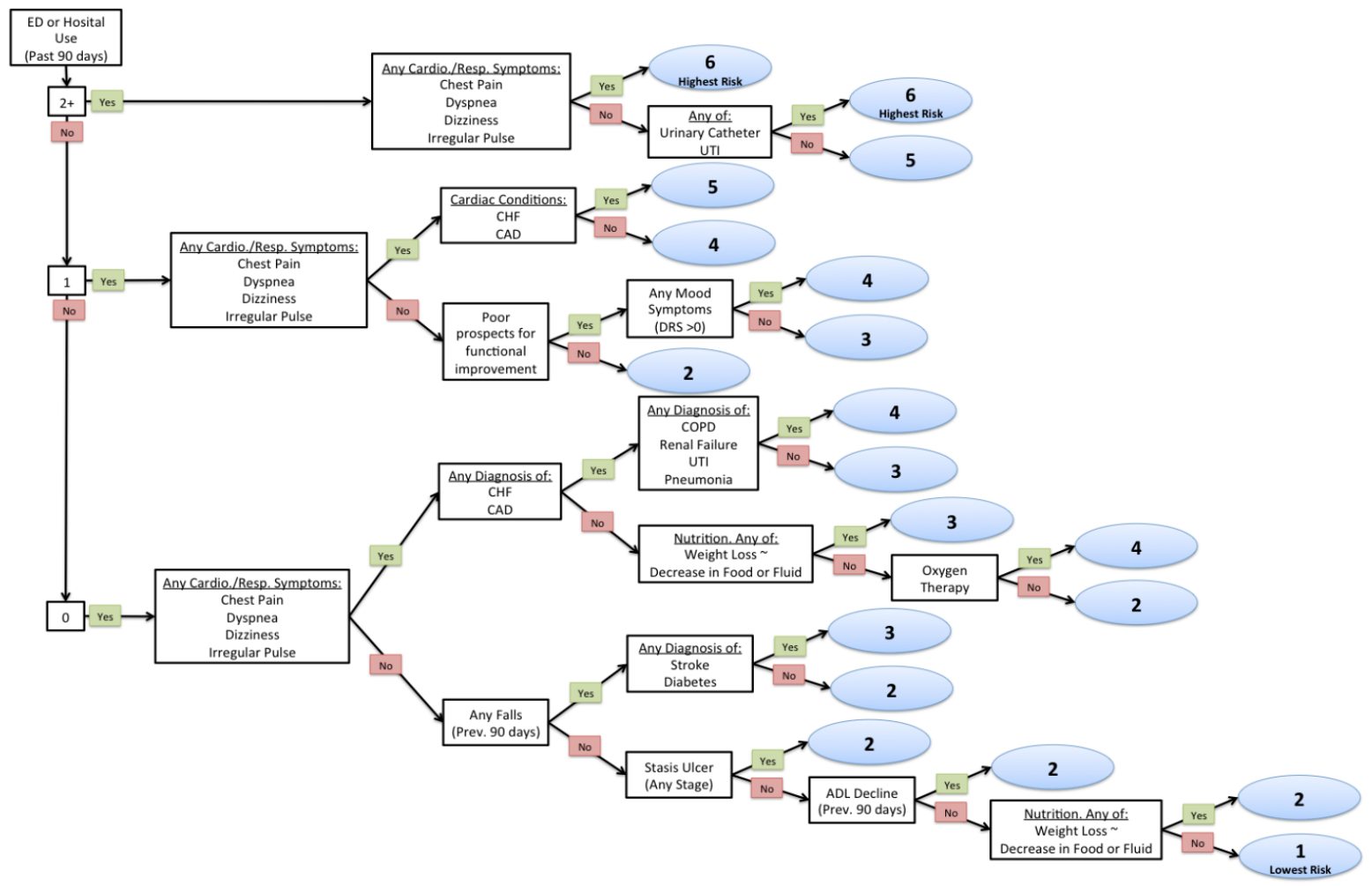

Figure 1: The logical algorithm for calculating the DIVERT scale (2)

Evidence about paramedic service use by older adults does not usually consider home care clients specifically, but studies have shown that older adults can account for a large percentage of patients transported. $(3,4)$ Home care populations are frequent users of the ED despite community-based care programs that are intended to maintain patients' health within their home. $(1,5,6)$ If designed and implemented effectively, these programs can reduce the risk of deterioration in health status or independence loss. $(7,8)$ For example, "Aging at home" programs have shown that patients can maintain independence at home without being transported to the ED with the assistance of a multi-disciplinary team.(9) However, complexities of multiple disease processes and difficulty navigating health care have been associated with use of EDs even when home care services are in place. (10) Many patients who visit an ED are not admitted to hospital, suggesting a lower acuity event and/or a preventable condition that may be amendable to earlier or alternative intervention. This assumes detection of modifiable risk factors is available. (11)

Community paramedicine is an example of an innovative approach in healthcare 
in response to pressures associated with an aging population and fiscal constraints.(11) Community paramedicine programs leverage the community of practice in which paramedics' work, often by expanding their scope of practice to identify needs and promote healthcare access across the continuum of care.(12) Tools used by paramedics to facilitate community based referrals for home care (13), community resources, or primary care $(14,15)$ are limited. Broadening the role of paramedics in healthcare suggests opportunities exist to explore how they might be better utilized in their community of practice which includes home care patient populations. Identifying patients who could benefit from home care or who may require enhanced home care services may be advantageous for paramedic services interested in engaging in preventative care through community paramedicine. Further case-finding tools are needed to assist community paramedics in care planning for patients and to facilitate information sharing between care providers. The DIVERT scale could be used to identify community dwelling older adults who are likely to use paramedic services for transport to an ED. The objective of this study was to investigate whether patients that were identified as being at higher risk for an ED visit based on their DIVERT scores were more likely to use paramedic services within 90 days of assessment when compared to clients that had lower DIVERT scores.

\section{Methods}

Overview

A retrospective cohort study using data derived from regularly collected administrative records was used to investigate paramedic service use by community dwelling older adults. Data about the home care patient population was collected that included risk of ED use (through DIVERT scores) and subsequent use of paramedic services (through mode of arrival) documented for ED visits that occurred after the assessment of risk. Data were tested to determine if DIVERT scores were predictive of paramedic service use. The research was approved by the Hamilton Integrated Research Ethics Board (\#1650).

\section{Setting}

Data were obtained from the Hamilton Niagara Haldimand Brant (HNHB) Local Health Integrated Network (LHIN) (including the former HNHB Community Care Access Centre (CCAC)). The HNHB region is situated in southern Ontario, Canada and is bordered to the east by the United States with Lake Ontario to the north, Lake Erie to the south and greater parts of the province to the west. The population of the region is approximately 1.4 million people who are served by 13 ED sites.

\section{Sample and Data Sources}

We complied a sample of all patients that received a home care assessment conducted by care coordinators for the former HNHB CCAC between April 1, 2015 and March 31, 2016. The home care assessment must have utilised the Residential Assessment Instrument for Home Care (RAI-HC) (16) assessment instrument to be considered. The RAI-HC is used by HNHB LHIN to determine eligibility for home care services. It includes items assessing function, cognition, medical history, social and demographic indicators (e.g., age, gender, living arrangement, educational level, geography), and items that inform risk scores and decision support tools (including DIVERT). Receiving a RAI$\mathrm{HC}$ assessment does not mean that an individual receives home care services, but it does identify risk factors at intake. If home care services are provided, these assessments are routinely repeated every 6 months to assess any changes in patient conditions. Care coordinators typically have a nursing background and assessments are typically completed in a patient's place of residence. The HNHB LHIN Integrated Decision Support provided access to data on hospital usage from the National Ambulatory Care Recording System (NACRS) that was linked to home care assessment data. Matched home care assessment and hospital utilisation data were provided for all patients in the cohort. One year of home care assessment records were provided with 15 months of records of corresponding to ED visits to the $13 \mathrm{ED}$ sites through data from the HNHB LHIN. NACRS data included acuity, disposition, mode of arrival (by land ambulance or other), and date and time information. Data were provided having been processed for 
quality assurance, matched, and de-identified (meaning that any data with missing fields were not included).

\section{Variables}

Independent variables available for analysis included DIVERT score and a variety of social and demographic information included in the RAI-HC assessment instrument. Age, gender, living arrangement, education level, and whether or not the individual lived in a rural location (as determined by postal code) were included as confounding variables that could influence the use of paramedic services. For example, individuals that lived in a rural location may display different tendencies in use of paramedic services than individuals that live in urban areas. Similarly, those that lived alone may use paramedic services differently than those who lived with a spouse or carer. The dependent variable of interest was whether clients used paramedic services for transport to hospital ED within 90 days of assessment or not.

\section{Analysis}

Preliminary review of the data was conducted to consider distribution of the variables. It was expected that distribution of the DIVERT scores within the cohort would not be evenly distributed as previous work showed that roughly $5 \%$ of the home care population are expected to have a score of 6 , but close to $70 \%$ of these clients are expected to visit the ED (2).

To test whether DIVERT scores were associated with increased likelihood of paramedic service use, a multivariate logistic regression model was devised. Adjusted odds ratios (ORs) were evaluated for models that had statistically significant independent variables (95\% confidence intervals that did not include the value 1.00). Plausible interaction terms were investigated for significance. Model performance was considered through a series of tests that considered goodness-of-fit, discriminatory performance, likelihood ratios, multicollinearity, and outliers. All analysis was performed using SAS version 9.4 (SAS Institute, Cary NC).

\section{Results}

Our final cohort was composed of 24283 patients (see Table 1). There was a higher proportion of female patients $61.67 \%(n=14973)$ and the mean age was 78 years $(\mathrm{SD}=13.8)$. Differences between the groups of patients that did and did not use paramedic services within the 90 days following assessment are evident. Our results show that $65.31 \%(n=6189)$ of all clients that visited the ED in the 90 days following assessment, did so using paramedic services. The mean length of time between assessment and first paramedic service use for these individuals was 29.31 days $(\mathrm{SD}=24.47)$. While the exposure of interest was use of paramedic services within 90 days of assessment, $18.17 \%(\mathrm{n}=3287)$ of those arriving at the ED within that period did not use paramedic services. A larger proportion of the group that used paramedic services made repeated ED visits over the course of the year $(87.27 \% ; n=5401$ compared to $33.37 \% ; n=6038)$.

DIVERT scores showed increasing likelihood of use of paramedic services from an OR of 1.40 (95\% confidence interval [1.25-1.58]) for a score of 2 up to an OR of 4.15 ([3.60-4.78]) for a score of 6 (when compared to a score of 1). A variety of combinations of models were tested using the variables shown in Table 2. While unadjusted ORs for age $(\mathrm{OR}=1.01$ [1.01-1.01]), gender (Male $\mathrm{OR}=1.23$ [1.16-1.31]), and living arrangement (with a carer) $(\mathrm{OR}=0.85$ [0.80-0.90]) were statistically significant, testing various combinations of their inclusion with DIVERT scores failed to improve model performance beyond modest levels. Interactions between terms also did not improve model performance. As a result, DIVERT scores without other variables pertaining to social factors was demonstrated as an appropriate predictor of paramedic service use.

The performance of the logistic regression model of DIVERT scores as a predictor of paramedic service use had an Area Under the Curve (AUC) of 0.61. Figure 2 illustrates the ROC curve for the model. Youden's Index was used to demonstrate discriminatory performance. The cut-point that maximizes Youden's Index is a probability of 0.28 which results in a sensitivity of 0.529 and a specificity of 0.638 (see Table 3). If an intervention were to be based on this cut-point, it would translate into 
$66.7 \%$ false positive rate and $20.2 \%$ false negative rate.

\begin{tabular}{|c|c|c|c|c|c|c|}
\hline Variable & Total & $\%$ & $\begin{array}{l}\text { Did not use } \\
\text { ambulance in } \\
90 \text { days } \\
\text { following }\end{array}$ & $\%$ & $\begin{array}{l}\text { Used } \\
\text { ambulance in } \\
90 \text { days } \\
\text { following }\end{array}$ & $\%$ \\
\hline n & 24283 & & 18094 & & 6189 & \\
\hline Visited ED within 90 days of assessment & 9476 & 39.02 & 3287 & 18.17 & 6189 & 100.00 \\
\hline Used ambulance at some point during the year & 9771 & 40.24 & 3582 & 19.80 & 6189 & 100.00 \\
\hline Visited ED more than once during year & 11439 & 47.11 & 6038 & 33.37 & 5401 & 87.27 \\
\hline Mean time between assessment and first ED & & & 119.09 & $( \pm 94.15)$ & 29.31 & $( \pm 24.47)$ \\
\hline DIVERT score of 1 & 3058 & 12.59 & 2609 & 14.42 & 449 & 7.25 \\
\hline DIVERT score of 2 & 6730 & 27.71 & 5422 & 29.97 & 1308 & 21.13 \\
\hline DIVERT score of 3 & 4669 & 19.23 & 3511 & 19.40 & 1158 & 18.71 \\
\hline DIVERT score of 4 & 5232 & 21.55 & 3708 & 20.49 & 1524 & 24.62 \\
\hline DIVERT score of 5 & 2997 & 12.34 & 1912 & 10.57 & 1085 & 17.53 \\
\hline DIVERT score of 6 & 1597 & 6.58 & 932 & 5.15 & 665 & 10.74 \\
\hline Male & 9310 & 38.34 & 6708 & 37.07 & 2602 & 42.04 \\
\hline Live with a caregiver & 12438 & 51.22 & 9462 & 52.29 & 2976 & 48.09 \\
\hline Attended post-secondary & 4999 & 20.59 & 3775 & 20.86 & 1224 & 19.78 \\
\hline Rural location & 3726 & 15.34 & 2806 & 15.51 & 920 & 14.87 \\
\hline Mean Age & \multicolumn{3}{|c|}{$78( \pm 13.8)$} & \multicolumn{2}{|c|}{$77.54( \pm 14.2)$} & $79.37( \pm 12.8)$ \\
\hline
\end{tabular}

Table 1. Summary of study cohort

\begin{tabular}{ll}
\hline Variable & Odds Ratio (95\% Confidence interval) \\
\hline DIVERT score of 1 & 1.00 \\
DIVERT score of 2 & $1.40(1.25-1.58)$ \\
DIVERT score of 3 & $1.92(1.70-2.16)$ \\
DIVERT score of 4 & $2.39(2.13-2.68)$ \\
DIVERT score of 5 & $3.30(2.91-3.74)$ \\
DIVERT score of 6 & $4.15(3.60-4.78)$ \\
& $($ Unadjusted) \\
Age & $1.01(1.01-1.01)$ \\
Male & $1.23(1.16-1.31)$ \\
Live with a Caregiver & $0.85(0.80-0.90)$ \\
Attended Post-Secondary & $0.94(0.87-1.01)$ \\
Rural & $0.95(0.88-1.03)$ \\
\hline
\end{tabular}

Table 2. Odds of using ambulance within 90 days of assessment

\begin{tabular}{lllll}
\hline Variable & Estimate & $\begin{array}{l}\text { Probability of } \\
\text { event }\end{array}$ & $\begin{array}{l}\text { Predicted at } \\
\mathbf{0 . 2 8} \text { cut point }\end{array}$ & Observed \\
\hline Intercept & -1.75 & & & \\
DIVERT score of 1 & & 0.15 & 0 & \\
DIVERT score of 2 & 0.34 & 0.19 & 0 & \\
DIVERT score of 3 & 0.65 & 0.24 & 0 & 1524 \\
DIVERT score of 4 & 0.87 & 0.30 & 5232 & 1085 \\
DIVERT score of 5 & 1.19 & 0.36 & 2997 & 665 \\
\hline DIVERT score of 6 & 1.42 & 0.42 & 1597 & \\
\hline
\end{tabular}

Table 3. Resulting probabilities from model 


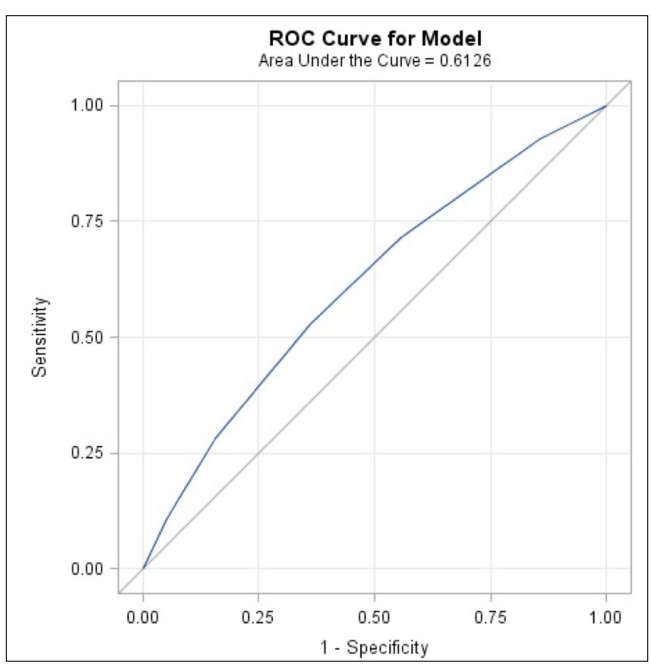

Figure 2: Receiver Operator Characteristic curve for logistic regression model of use of paramedic services predicted by DIVERT scores.

\section{Discussion}

The results demonstrate that DIVERT scores are predictive of paramedic service utilization by home care patients in the 90 days following RAI-HC assessment and validate application of DIVERT scores to predict the use of paramedic services as a mode of arrival for an ED visit. DIVERT was developed to evaluate levels of risk for home care clients visiting an ED but without considering means of transportation to the ED. The DIVERT scores presented for this cohort overall are relatively similar to previous findings (2), with the largest number of clients having a score of 2 and the fewest having a score of 6 . Other variables reflecting social factors were tested that could have an association with access to other means of transportation that may influence a patient's decision to call emergency services. Inclusion of these variables in the model building process did not improve the results achieved from DIVERT scores alone.

One of the challenges associated with case-finding tools is with respect to the differences between the sexes. The findings of the Paramedics assessing Elders at Risk for Independence Loss (PERIL) Study indicated that identification of male patients was significant as it related to adverse outcomes.(13) Known characteristics about male mortality suggest that gender differences are likely. We found that males had statistically significant odds for use of an ambulance within 90 days of home care assessment. Similarly, increased odds were noted for age and decreased odds for those that lived with a carer. We suspect that female clients who live with a carer are less likely to use paramedic services than male clients or those who do not live with a carer. However, exploring our assumption by investigating the independent variables at our disposal and any interactions between these terms, did not improve model performance beyond the most modest levels.

Future work can look at the association between temporal factors and subsequent ED utilization. For example, those that used an ambulance to get to the ED had an average time of use following assessment of less than a month (29.31 days) compared to almost four months (119.09 days) for those that did not use an ambulance. Survival analysis could provide further insight into these differences with a goal of tailoring provision of care to a "just-in-time" model. Forecasting use of emergency services involves considering their stochastic nature. Our investigation focused on information that may be available pre-emptively through the standardized RAI-HC assessment instrument. For that reason, we did not include information regarding the nature of ED visits such as acuity level or time of visit. Others have noted the relevance of off-hours use (defined as outside of regular business hours of Monday to Friday between 9:00am and $5: 00 \mathrm{pm})$ as informative with respect to patient profiles.(17,18) Investigation of our dataset revealed that time of use may be worthy of further exploration as more clients used paramedic services outside of regular business hours than within regular business hours. 
The results indicate that consideration of DIVERT scores could be a valuable risk assessment tool for paramedics to use with community dwelling older adults. The discriminatory performance of our logistic regression model demonstrated that it would be feasible to classify all patients with a DIVERT score of 4 or greater as being likely to use an ambulance. Using our cohort as an example would mean that 9,826 patients would receive this prediction. Further study is required to explore the utility of such a classification, to investigate actual utilization by pre-identified patients, and to consider preventative interventions.

One of the limitations of using administrative data is that we cannot comment on whether social factors such as living arrangement or living in a rural location were influential at the time of paramedic service use. Further investigation of these social factors and consideration of aspects of social isolation and carer resiliency as they influence the use of paramedic services is warranted.

\section{Conclusion}

The DIVERT scale is used to identify home care clients at greatest risk for using the ED. The results from our investigation of indicators of paramedic service use by community dwelling older adults demonstrates that the DIVERT Scale is suitable for identifying clients likely to use an ambulance provided by paramedic services for transport to the ED within 90 days. Future work needs to identify interventions that are suitable for addressing the needs of these clients and exact clinical situations of use.

\section{References}

1. Costa AP, Harkness K, Haughton D, Heckman GA, McKelvie RS. Risk of emergency department use among community-dwelling older adults : a review of risk factors and screening methods. Clin Pract [Internet]. 2014 Dec [cited 2016 Dec 15];11(6):763-76. https://doi.org/10.2217/cpr.14.66

2. Costa AP, Hirdes JP, Bell CM, Bronskill SE, Heckman GA, Mitchell L, et al. Derivation and validation of the detection of indicators and vulnerabilities for emergency room trips scale for classifying the risk of emergency department use in frail community-dwelling older adults. J Am Geriatr Soc. 2015;63(4):763-9. https://doi.org/10.1111/jgs.13336.

3. Singal BM, Hedges JR, Rousseau EW, Sanders AB, Berstein E, McNamara RM, et al. Geriatric patient emergency visits. Part I: Comparison of visits by geriatric and younger patients. Ann Emerg Med [Internet]. 1992 Jul [cited 2016 Dec 15];21 (7):802-7.

4. Munjal KG, Silverman RA, Freese J, Braun JD, Kaufman BJ, Isaacs D, et al. Utilization of emergency medical services in a large urban area: description of call types and temporal trends. Prehosp Emerg Care [Internet]. 2011;15(3):371-80. https://doi.org/10.3109/10903127.2011.561403

5. Sinha SK, Bessman ES, Flomenbaum N, Leff B. A systematic review and qualitative analysis to inform the development of a new emergency departmentbased geriatric case management model. Ann Emerg Med. 2011;57(6):672-82. https://doi.org/10.1016/j.annemergmed.2011.01.021.

6. Coe AB. Identifying Problems during Transitions of Care and Reasons for Emergency Department Utilization in Community-Dwelling Older Adults. 2015;290. (Thesis; https://scholarscompass.vcu.edu/etd/4022/)

7. Morris JN, Howard EP, Steel K, Schreiber R, Fries BE, Lipsitz LA, et al. Predicting risk of hospital and emergency department use for home care elderly persons through a secondary analysis of cross-national data. BMC Health Serv Res [Internet]. 2014;14(1):519. https://doi.org/10.1186/s12913-014-0519-z

8. Kessler C, Williams MC, Moustoukas JN, Pappas C. Transitions of Care for the Geriatric Patient in the Emergency Department. Clin Geriatr Med [Internet]. 2013 [cited 2016 Dec 15];29:49-69. https://doi.org/10.1016/j.cger.2012.10.005

9. Ruest M, Stitchman A, Day C. Evaluating the impact on 911 calls by an in-home programme with a multidisciplinary team. Int Paramed Pract. 2012;1(4):125-32. https://doi.org/10.12968/ippr.2012.2.2.41

10. Pines JM, Mullins PM, Cooper JK, Feng LB, Roth KE. National trends in emergency department use, care patterns, and quality of care of older adults in the 
United States. J Am Geriatr Soc. 2013;61(1):12-7. https://doi.org/10.1111/ jgs. 12072.

11. Kellermann AL. Emergency medicine and public health: Stopping emergencies before the 9-1-1 call. Acad Emerg Med. 2009;16(11):1060-4.

12. CSA Group. Community Paramedicine: Framework for program development. Toronto, ON: CSA Group; 2017.

13. Lee JS, Verbeek PR, Schull MJ, Calder L, Stiell IG, Trickett J, et al. Paramedics assessing Elders at Risk for Independence Loss (PERIL): Derivation, Reliability and Comparative Effectiveness of a Clinical Prediction Rule. CJEM [Internet]. 2016;18(02):121-32. https://doi.org/10.1017/cem.2016.14

14. Agarwal G, McDonough B, Angeles R, Pirrie M, Marzanek F, Mcleod B, et al. Rationale and methods of a multicentre randomised controlled trial of the effectiveness of a Community Health Assessment Programme with Emergency Medical Services (CHAP-EMS) implemented on residents aged 55 years and older in subsidised seniors' housing b. BMJ Open [Internet]. 2015;5(6):e008110 e008110. https://doi.org/10.1136/bmjopen-2015-008110

15. Agarwal G, Angeles R, Pirrie M, Marzanek F, McLeod B, Parascandalo J, et al. Effectiveness of a community paramedic-led health assessment and education initiative in a seniors' residence building: the Community Health Assessment Program through Emergency Medical Services (CHAP-EMS). BMC Emerg Med [Internet]. 2016;17(1):8. https://doi.org/10.1186/s12873-017-0119-4

16. Morris JN, Fries BE, Bernabei R, Steel K, Ikegami N, Carpenter I, et al. RAIHome Care ( RAI-HC ) Manual. Second. interRAI Corporation; 2002.

17. Cantwell K, Morgans A, Smith K, Livingston M, Spelman T, Dietze P. Time of Day and Day of Week Trends in EMS Demand. Prehospital Emerg Care [Internet]. 2015;19(3):425-31. https://doi.org/10.3109/10903127.2014.995843

18. Scott J, Strickland AP, Warner K, Dawson P. Describing and predicting frequent callers to an ambulance service: analysis of 1 year call data. Emerg Med J [Internet]. 2013;1-7. https://doi.org/10.1136/emermed-2012-202146 